\title{
Bacterial meningitis associated with lumbar drains: a retrospective cohort study
}

\author{
William M Coplin, Anthony M Avellino, D K Kim, H Richard Winn, M Sean Grady
}

\begin{abstract}
Objectives-The infective potential of lumbar drainage is an important topic deserving particular study. The aetiology, incidence, and clinical findings associated with bacterial meningitis are described in patients having continuous lumbar CSF drainage to treat communicating hydrocephalus after subarachnoid haemorrhage or CSF leaks after traumatic dural rents.
\end{abstract}

Methods-Retrospective review of the records of patients with a positive CSF bacterial culture who underwent lumbar drain placement over a 39 month period. Results-Thirteen cases of bacterial meningitis occurred subsequent to the use of 312 lumbar drain kits (4.2\%). All meningitic patients had CSF pleocytosis, but not all had peripheral leukocytosis. Fever, peripheral leukocytosis, and CSF pleocytosis did not help to differentiate the presence of bacterial meningitis from other infections. Eight patients had prior CSF drainage procedures, including ventriculostomy $(n=5)$ or lumbar drain $(n=5)$ placements; two patients received both procedures. Six of 13 patients developed their CSF infection within 24 hours of lumbar drain insertion. Six of 13 patients developed meningitis while receiving antibiotics for other reasons.

Conclusions-External lumbar drainage seems to carry a low risk of infectious meningitis and offers a safe alternative to ventriculostomy or serial lumbar punctures. Antibiotics do not seem to protect completely against developing the infection. The infection happens most often with skin organisms. The meningitis often appears within 24 hours after lumbar drain placement. Daily CSF samples should include bacterial cultures but cell counts may not offer any additional useful information in diagnosing the complication. Lumbar drain insertion and management need not be confined to the intensive care unit.

(F Neurol Neurosurg Psychiatry 1999;67:468-473)

Correspondence to: Dr William M Coplin, Departments of Neurology and Neurological Surgery,

Wayne State University, 4201 St Antoine, 8D-UHC,

Detroit, MI 48201, USA.

Telephone 001313577

1242; fax 0013137454216 ;

email

wcoplin@med.wayne.edu

Received 6 July 1998 and in revised form

25 March 1999

Accepted 24 April 1999 a temporising measure in treating communicating hydrocephalus caused by aneurysmal subarachnoid haemorrhage ${ }^{12}$ and is one treatment for correcting CSF leaks (spinal or cranial). Similarly to ventriculostomy, there is a
Keywords: cerebrospinal fluid; lumbar drainage; meningitis risk of meningitis to consider when using this modality, and the infective potential of lumbar drainage is an important topic deserving particular study. We conducted a retrospective cohort study of patients with positive CSF cultures after lumbar drain placement to ascertain the possible aetiologies of meningitis. Our objectives included defining the incidence of meningitis after lumbar drain placement, describing the association of positive CSF cultures with the clinical signs and laboratory values considered diagnostic of meningitis, and describing potential risk factors for contracting lumbar drain associated meningitis.

\section{Patients and methods}

PATIENT SELECTION

The method of patient selection was identification of all cases of bacterial meningitis. Cases established by culture were identified from a list of all positive CSF cultures at Harborview Medical Center, Seattle, WA, USA between June 1992 and December 1995. Hospital charts were then screened for those who received a lumbar drain. We reviewed in detail the records of those patients who underwent lumbar drainage. Patients' charts were used to confirm information from other data sources (for example, microbiology laboratory records). Diagnoses treated by lumbar drains included communicating hydrocephalus after subarachnoid haemorrhage and traumatic cranial CSF leak. The degree of hydrocephalus or CSF leak and the patient's clinical condition determined the duration of drainage. We obtained our denominator (the number of lumbar drain placements over this time) from intrahospital usage of lumbar drain kits. The number of kits used was likely higher than the number of patients treated as there were patients who had more than one drain placed during their stays in hospital. The Human Subjects Review Committee of the University of Washington approved this research activity.

\section{LUMBAR DRAIN TECHNIQUE}

After shave (if necessary), skin preparation (povidone-iodine solution), and draping of the lumbosacral area, 1\% lidocaine was infiltrated between the palpated spinous processes of the lower lumbar vertebrae. A $16 \mathrm{G}$ Tuohy spinal needle was advanced until it punctured the meninges. A CSF sample was sent for routine studies (cell count and differential, glucose, protein, gram stain, and culture). The lumbar drain was threaded into the subarachnoid space. The needle was withdrawn and the drain attached to a drainage bag. A silk tie secured the adapter connecting the intraspinal catheter 
and the external drainage system. Sterile gauze dressed the skin wound. A transparent adhesive dressing covered the external part of the intraspinal catheter to prevent dislodgment. Patients did not receive specific antibiotic prophylaxis for the procedure.

Samples of CSF were obtained from a sampling port along the drainage catheter. This port lies just distal to the junction of the indwelling catheter and its connection to the remainder of the drainage system leading to the collection bag. This site has a three way port that was closed to the drainage bag side for sampling. Also, before sampling, the membrane over the sampling port was swabbed with a povidone-iodine solution that was allowed to dry completely before a sterile needle was introduced through the membrane to obtain the CSF sample.

CLINICAL AND EPIDEMIOLOGICAL DATA

ABSTRACTION

The following demographic data came from the charts of patients who had a positive bacterial CSF culture and a lumbar drain: age, hospital admission date, admitting neurological diagnosis (for example, subarachnoid haemorrhage, traumatic CSF leak), and if the patient had a history of diabetes mellitus. Operations were recorded (including if the surgeon entered the ventricles) as well as neurological instrumentation (for example, placement of intracranial pressure monitors or ventriculostomies). Other clinical data were gathered-namely, other positive CSF cultures (lumbar puncture, ventriculostomy); other microbiological culture results (for example, blood, urine, sputum, wound); CSF cell counts for 48 hours before and after drawing the culture positive sample; temperatures for 48 hours before and after drawing the culture positive sample; signs of drain site infection (for example, leaking CSF, purulence, erythema, induration); duration of lumbar drainage; peripheral white blood cell count; intracranial and CSF opening pressures; antibiotic use before identification of infection; CT findings (for example, presence of hydrocephalus or intraventricular or intraparenchymal haemorrhage); and most recent chest radiography findings.

The location in the hospital at which the physician placed the lumbar drain (for example, intensive care unit, intermediate care unit, or ward), and on which ward the patient was at the time of positive CSF culture were noted. A nursing documentation sheet was used to determine dressing and drainage system change frequencies; the amount, colour (for example, xanthachromic $v$ colourless), and clarity (for example, cloudy $v$ clear) of CSF drained; and the vertical position of the drain in relation to the patients' head. This record included the presence of CSF leaking from the drain insertion site and drain manipulations. The profile of the culture positive CSF (cell count and differential, glucose, protein, and gram stain) was recorded. Standard lumbar drain protocol included daily collection of CSF samples for cell count and differential, glucose, protein, Gram stain, culture, and sensitivities of organisms isolated (if any).

DEFINING VARIABLES

Meningitis associated with lumbar drains was defined before starting the review. Among other variables, the review sought to explore the possible association of leukocytosis, fever, and pleocytosis (predominance of segmented neutrophils) with meningitis diagnosed by positive CSF culture. These definitions were derived from a prior study of infections related to ventriculostomy. ${ }^{3}$ The incidence of ventriculostomy infections was beyond the scope of this study.

The diagnosis of meningitis associated with lumbar drains required the following criteria: (1) no previous meningitis before lumbar drain insertion; (2) sterile CSF culture at the time of lumbar drain insertion; (3) continuous lumbar drainage $\geqslant 24$ hours before the positive culture was aspirated; and (4) positive culture of a CSF specimen collected from the lumbar drain or from a lumbar puncture.

Fever was defined as a temperature $\geqslant 38.5^{\circ} \mathrm{C}$. Association of fever with meningitis required the following criteria: (1) fever within 48 hours of the positive CSF culture; (2) no other concurrent infection; (3) if the patient had fever within 48 hours before the positive CSF culture, then a temperature rise $\geqslant 0.6^{\circ} \mathrm{C}$ within 48 hours of the positive CSF culture; or (4) fever lasting for 3 days.

Peripheral leukocytosis was defined as $>10000$ white blood cells (WBC) $/ \mathrm{mm}^{3}$ of blood. The attribution of the leukocytosis to meningitis required: (1) leukocytosis occurring within 48 hours of the positive CSF culture; (2) no other concurrent infection; (3) if the WBC count was $>1000048$ hours before the positive CSF culture, then an increase in WBC $\geqslant 1000 /$ $\mathrm{mm}^{3}$ within 48 hours of the positive culture; or (4) leukocytosis lasting for 3 days.

Pleocytosis in CSF was defined as a CSF WBC count $\geqslant 11$ cells $/ \mathrm{mm}^{3}$. Attributing CSF pleocytosis to acute bacterial meningitis required: $(1) \geqslant 50 \%$ of the WBC were segmented neutrophils; (2) pleocytosis occurring within 48 hours of the positive CSF culture; (3) if there was pleocytosis 48 hours before the positive culture, then an increase of $\geqslant 50 \mathrm{WBC} / \mathrm{mm}^{3}$ within 48 hours of the positive culture; or (4) pleocytosis lasting at least 3 days.

\section{DATA ANALYSIS}

Data were entered into a computerised database (SPSS for Macintosh, version 6.1.1, SPSS Inc, Chicago, IL, USA) University of Washington computer resources were used to analyse the data. Analysis consisted of Fisher's exact test (two sided) along with the descriptive statistics.

\section{Results}

The initial review identified 24 patients with positive CSF cultures and a case record of lumbar drain insertion. Excluded from detailed review were 11 of these 24 patients whose CSF cultures were considered to be plate contamination (for example, colony count of $1 ; n=8$ ), 
Table 1 Clinical characteristics of 13 patients with lumbar drain-associated bacterial meningitis

\begin{tabular}{lllllll}
\hline Patient & Age (y) & $\begin{array}{l}\text { Neurological } \\
\text { problem }\end{array}$ & $\begin{array}{l}\text { Prior } \\
\text { surgeries }\end{array}$ & $\begin{array}{l}\text { Drainage } \\
\text { duration }\end{array}$ & $\begin{array}{l}\text { Concurrent } \\
\text { antibiotics }\end{array}$ & Organism(s) \\
\hline 1 & 47 & SAH & None & 7 & No & Kpneumoniae \\
2 & 53 & SAH & V, C & 3 & Yes & CNS \\
3 & 64 & SAH & V, C & 4 & Yes & CNS \\
4 & 34 & SAH & C & 1 & No & CNS \\
5 & 69 & see below† & C, L & 9 & No & Paeruginosa \\
6 & 53 & SAH & V, C & 3 & No & Actinobacter calcoaceticus \\
7 & 24 & BSF & None & 1 & No & group A Streptococcus \\
8 & 84 & SAH & None & 1 & No & CNS \\
9 & 48 & SAH & V, C & 1 & No & S aureus \\
10 & 61 & SAH & V, C $\subseteq$ & 2 & Yes & CNS, Corynebacterium \\
& & & M & & & species \\
11 & 25 & BSF & M & 1 & Yes & Enterobacter cloacae \\
12 & 42 & AVM & R, L & 1 & Yes & CNS \\
13 & 50 & SAH & None & 6 & Yes & S aureus \\
\hline
\end{tabular}

${ }^{\star}$ This number does not count the day of lumbar drain placement but does count the day the culture positive sample was taken.

†Patient 5 had a CSF leak after sinus surgery.

$\ddagger$ All patients had prior intracranial pressure monitors, except this one.

§Two separate craniotomies.

$\mathrm{SAH}=$ subarachnoid haemorrhage; $\mathrm{BSF}=$ basilar skull fracture; $\mathrm{AVM}=$ arteriovenous malformation; $\mathrm{V}=$ =ventriculostomy; $\mathrm{C}=$ aneurysm clipping; $\mathrm{L}=\mathrm{CSF}$ leak repair; $\mathrm{M}=$ repair of mandibular fracture; $\mathrm{R}=$ resection; $\mathrm{CNS}=$ coagulase negative staphylococci.

Table 2 Relation of fever, peripheral leukocytosis, and CSF pleocytosis to lumbar drain associated bacterial meningitis

\begin{tabular}{llllll}
\hline & \multicolumn{2}{l}{$\begin{array}{l}\text { Patients without other infection } \\
(n=8)\end{array}$} & $\begin{array}{l}\text { Patients with other infection } \\
(n=5)\end{array}$ & \\
\cline { 2 - 5 } & Sign present & Sign absent & Sign present & Sign absent & p Value * \\
\hline Fever & 4 & 4 & 4 & 1 & 0.56 \\
Peripheral leukocytosis & 6 & 2 & 3 & 2 & 1.00 \\
CSF pleocytosis & 8 & 0 & 5 & 0 & 1.00 \\
\hline
\end{tabular}

^By Fisher's exact test (two sided).

and those who had a proved prior CSF infection $(n=3)$. By the study's criteria, bacterial meningitis complicated 13 of 312 lumbar drains $(4.2 \%)$ inserted during the study period. The age range of patients with meningitis was 24 to 84 years (median 50). Table 1 presents demographic data for the 13 patients. Five patients had previous ventriculostomy. Twelve patients had a prior intracranial pressure monitor. Three patients had subarachnoid haemorrhage without angiographic identification of a source. Eight patients had a total of 11 intracranial operations; seven of these patients underwent eight aneurysm clippings after presenting with subarachnoid haemorrhage; two patients required repair of CSF leaks; one patient had resection of an arteriovenous malformation. One patient's only surgery was repair of a mandible fracture. Three patients had cranial CSF leaks before lumbar drain insertion. Not all patients had daily CSF samples sent from their lumbar drains.

CULTURE DATA

A positive CSF culture aspirated from the lumbar drain confirmed all cases. Table 1 shows the culture results. The most common organisms isolated were coagulase negative staphylococci $(\mathrm{n}=7 / 13)$. Eleven of the 15 isolates found in the 13 patients were gram positive organisms. Four patients had meningitis from a gram negative bacillus. One patient had CSF infection with multiple skin organisms. Two patients had positive cultures from CSF specimens collected over 3 consecutive days; one patient had positive cultures from CSF specimens collected over 4 consecutive days. One patient had positive cultures both from lumbar drain and lumbar puncture samples; the same organism grew from both CSF samples.

Other infections (bacteraemia, pneumonia, or urinary tract or wound infections) occurred in five of 13 cases of lumbar drain associated meningitis. Five of the patients became bacteraemic within 48 hours of the positive CSF culture sample; only one of these patients developed bacteraemia with an organism with the same antibiotic sensitivity pattern as that isolated from CSF (coagulase negative staphylococci). Five patients had positive sputum cultures within 48 hours of the positive CSF culture; three of these grew mixed flora and the other two grew organisms different from the one causing meningitis. Only one of the 13 patients met clinical criteria for the diagnosis of pneumonia (leukocytosis, fever, purulent sputum, and new progressive chest infiltrate on radiography). Four patients had positive urine cultures within 48 hours of drawing the positive CSF sample; in one of these cases the same organism grew from blood and from CSF (coagulase negative staphylococci). One patient had a positive culture taken from the lumbar drain insertion site; this sample grew two organisms, one of which was the same as that causing the meningitis (coagulase negative staphylococci). One patient had a positive culture of an intravenous catheter tip; this organism was different from the one associated with meningitis. In one patient the culture of the intracutaneous part of the lumbar drain resulted in growth of the same organisms as that from CSF (Corynebacterium minutissimum and $S$ epidermidis).

ASSOCIATION OF MENINGITIS WITH CSF PLEOCYTOSIS, FEVER, AND PERIPHERAL LEUKOCYTOSIS

Table 2 shows the relation of fever, peripheral leukocytosis, and CSF pleocytosis with 13 cases of bacterial meningitis. Fever and peripheral leukocytosis are not specific clinical signs for bacterial meningitis associated with lumbar drainage. All of the patients with meningitis had CSF pleocytosis. Although the presence of CSF pleocytosis seemed to be an insensitive marker for bacterial meningitis, a rising CSF polymorph count may have heralded the infection. Of the seven patients with meningitis for whom preinfection WBC counts in CSF were available, all seven had CSF WBC counts>11 cells $/ \mathrm{mm}^{3}$; these counts increased to as high as 2208 cells $/ \mathrm{mm}^{3}$.

POSSIBLE RISKS FOR CONTRACTING BACTERIAL MENINGITIS ASSOCIATED WITH LUMBAR DRAINS Table 3 describes the association of several putative risks for contracting bacterial meningitis associated with lumbar drains. A major problem of this uncontrolled analysis is that few infections occurred over these 39 months; therefore, the associations investigated were not found to be significant.

There were some potential associations between continuous lumbar CSF drainage and the subsequent development of bacterial meningitis. Table 1 shows that bacterial meningitis 
Table 3 Description of putative risks for developing lumbar drain associated bacterial meningitis

\begin{tabular}{lc}
\hline Risk & $\begin{array}{l}\text { No of patients } \\
(\%)\end{array}$ \\
\hline $\begin{array}{l}\text { Neurological diagnosis (based on CT) } \\
\text { Subarachnoid haemorrhage }\end{array}$ & $9(69)$ \\
Intraparenchymal haemorrhage & $3(23)$ \\
Intraventricular haemorrhage & $7(54)$ \\
Hydrocephalus & $8(61)$ \\
Underlying diabetes & $1(7)$ \\
Neurosurgical operation & $8(61)$ \\
Ventricles entered & $0(0)$ \\
Drain placed out of intensive care unit & $6(46)$ \\
Antibiotics & $6(46)$ \\
Opening CSF pressure $\geqslant 20$ mm Hg & $4(57)^{\star}$ \\
Intracranial pressure $\geqslant 20$ mm Hg & $3(23)$ \\
Drain duration $>5$ days & $3(23)$ \\
Problem/manipulation & \\
Leaks & $5(38)$ \\
Disconnections & $1(7)$ \\
System changes & $7(54)$ \\
Dressing changes & $13(100)$ \\
Gross blood & $0(0)$ \\
Prior ventriculostomy & $5(38)$ \\
Prior intracranial pressure monitor & $12(92)$ \\
\hline
\end{tabular}

Risk set $=13$.

${ }^{\star}$ Data not available for 6 patients; this risk set $=7$.

occurred in 10 of our 13 cases by day 4 , and all cases appeared by day 9 of continuous lumbar drainage; however, six of 13 infections occurred with catheters in place for 1 day. As outlined in table 3, intraventricular haemorrhage and subarachnoid haemorrhage had $>50 \%$ coincidence in our cohort, and these may be important, but significance could not be determined because of our small sample size.

The meningitis was treated in all patients, except for one whose family decided to withdraw supportive measures. This patient was the only one with meningitis associated with lumbar drain as a cause of death. Otherwise, the drain was removed, appropriate intravenous antibiotics started, and, later, a decision made as to whether to reinstitute lumbar drainage.

\section{Discussion}

INCIDENCE AND RISK OF BACTERIAL MENINGITIS ASSOCIATED WITH LUMBAR DRAINS

Smaller series have considered the meningitis risk with lumbar drain use. One reported 7 days of lumbar drainage in 39 patients to treat CSF leaks after skull base surgeries; none received antibiotic prophylaxis. Ten developed infectious meningitis $(25.6 \%)$. The authors concluded that concomitant antibiotic prophylaxis is unnecessary. ${ }^{4}$ In a series reviewing seven years of experience with continuous lumbar CSF drainage for 48 patients with CSF leaks at operative sites (11 of 48) or CSF rhinorrhea (37 of 48), none developed infectious meningitis. ${ }^{5}$ In our cohort, the incidence of lumbar drain bacterial meningitis was $4.2 \%$, which included nine patients with subarachnoid haemorrhage and four patients with traumatic or iatrogenic CSF leaks. From our cohort and others, it seems that placement of lumbar drains is safe, with minimal risk of infection.

The incidence of bacterial meningitis of $4.2 \%$ in this series is as might be predicted from the ventriculostomy infection data. Others have found a wide range of CSF infection rates per ventriculostomy placement $(0 \%$ of $11^{6}$ and $30,{ }^{7} 4.5 \%$ of $65,{ }^{8} 8.9 \%$ of $213,{ }^{3}$ and $10.3 \%$ of $29^{9}$ ). Another series compared tunnelled ventriculostomy (10\% infection rate for 70 placements) with the standard Rickham reservoir (27\% infected after 66 placements). ${ }^{10}$ In a comparison of the effect of antibiotic prophylaxis, others found infection rates of $9 \%$ (44 placements with antibiotics) to $27 \%$ (26 placements without antibiotics). ${ }^{11}$ A prospective study did not find a reduced risk with antibiotic use. ${ }^{3}$ Our rate may differ from that of the ventricular CSF drainage studies because of the different technique or definitions. Other investigators have defined meningitis associated with CSF drainage differently in studying ventricular drainage systems. ${ }^{369-11}$

As to possible risk factors, most patients had subarachnoid haemorrhage (nine of 13) or intraventricular haemorrhage (seven of 13), but not intraparenchymal haemorrhage (present in three of 13). Although four ventriculostomy series have discussed neurological diagnosis ${ }^{31011}$ only one explored the association of intraventricular haemorrhage with CSF infection. ${ }^{3}$ Blood products may be a good medium for culture and may block CSF resorption, preventing clearance of debris.

Patients with intracranial hypertension or intraventricular haemorrhage may require prolonged CSF drainage. We found intracranial hypertension at hospital presentation in only three of 13 patients; lumbar drain placement CSF opening pressure was raised in four of seven patients for whom these data were available. Others have noted an association between intracranial hypertension and the development of ventriculostomy related meningitis, independent of drainage duration. ${ }^{3}$

Neurosurgical procedures are reported to precede ventriculostomy related meningitis cases, ${ }^{12}$ and intracranial surgery preceded lumbar drain insertion in eight of our 13 patients, but none had their ventricles breached in that surgery.

Ten of 13 patients contracted meningitis within 4 days of lumbar drain placement, and six did so within the first day of lumbar drainage. Five series have considered the association of duration of ventriculostomy with the incidence of meningitis, ${ }^{3}$ 10-12 $^{12}$ two describing a direct relation between risk of infection and duration of drainage. The present data do not support an optimal interval for routine lumbar drain change. In patients requiring continued drainage, a fresh drain can be inserted or serial lumbar puncture can be used. Five of the 13 patients underwent prior ventriculostomy; 12 received an intracranial pressure monitor, and five patients were treated with a previous (uninfected) lumbar drain.

Only one patient had disconnection of the external CSF drainage system. Others have described a relation between infection and ventriculostomy irrigation. ${ }^{311}$ Opening the system can allow bacterial passage into the subarachnoid space. 
ASSOCIATION WITH OTHER INFECTIONS

This study defined bacterial meningitis as a positive culture from a CSF sample withdrawn from a lumbar drain. Eight of the initially identified 24 patients did not have lumbar drain associated meningitis, but had contaminated CSF samples. Additionally, there were three patients with bacterial meningitis before insertion of the lumbar drain.

Five of 13 patients had an infection other than meningitis, as shown in table 2 . The lack of a relation between clinical findings and having meningitis versus another infection may reflect the small sample size.

Having pleocytosis was not statistically related to having meningitis. All patients had CSF pleocytosis in response to culture established infections. Given the potentially serious consequences of delayed diagnosis, it is of concern that CSF cell counts do not offer a reliable method of diagnosis before culture results are available. We did not ascertain the likelihood of the converse - that is, patients with pleocytosis without culture proved infection. Pleocytosis could result from non-infectious meningeal inflammation, such as to a foreign body (the lumbar drain). Others have shown CSF pleocytosis after ventriculostomy with sterile cultures. ${ }^{3813}$ Thus, pleocytosis alone may not suffice in diagnosing lumbar drain associated bacterial meningitis, and the diagnostic "gold standard" remains CSF culture obtained from either the drain or from lumbar puncture. We cannot comment further on the subject of a rising polymorph count in the CSF as a harbinger of culture proved meningitis. Clinical judgment must dictate individual decisions regarding drain removal and initiation of therapy before having culture results.

RELEVANCE AND CLINICAL SIGNIFICANCE TO LUMBAR DRAIN USE

There are three main temporising measures for patients requiring continual CSF drainage: serial lumbar puncture, lumbar drainage, and ventriculostomy. Hydrocephalus occurs in $20 \%-31 \%$ of patients with subarachnoid haemorrhage, $40 \%$ improving within 24 hours without intervention. ${ }^{14}{ }^{15}$ Others need their communicating hydrocephalus treated only with transient CSF drainage, either by continuous external catheter or by serial lumbar puncture. Using long tunnelled ventriculostomy, and giving only perioperative antibiotic prophylaxis, the incidence of infectious meningitis is reported to be $4 \%$, similar to the rate in the present study. ${ }^{16}$ Using serial lumbar puncture to treat symptomatic hydrocephalus after subarachnoid haemorrhage, none of 17 patients developed infectious meningitis. ${ }^{15}$

Serial lumbar puncture carries a risk of infection and causes repeated patient discomfort. Infection complicating lumbar puncture can happen after diagnostic or therapeutic drainage of $\mathrm{CSF},{ }^{17-24}$ epidural anaesthesia, ${ }^{25}$ myelography, ${ }^{26}{ }^{27}$ or intrathecal infiltration (for example, chemotherapy administration). Causative organisms include Staphylococcus aureus, and streptococci ( $S$ pneumoniae, $S$ salivarius, etc). ${ }^{17} 182426$ There are no clear data concerning the incidence of meningitis per diagnostic CSF examination.

A patient's own infected blood contaminating a spinal needle can directly introduce bacteria. ${ }^{28} \mathrm{~A}$ retrospective series found an incidence of meningitis of $2.1 \%$ (three of 140) in patients receiving lumbar puncture while bacteraemic. This was not a significant difference from the expected $0.8 \%$ (seven of 924) incidence of meningitis in patients similarly bacteraemic who did not receive lumbar puncture. $^{29}$

\section{POTENTIAL ERROR}

The denominator in our record review (drain kits used) may not accurately reflect the number of patients receiving drains or the actual number of drains placed. For practical reasons, we were unable to look at all the patients treated with lumbar drainage. Our denominator assumes that hospital inventory records are accurate and that all kits are actually used for the specific purpose (and none lost or discarded because of contamination or technical difficulties, etc). It is likely that some patients had more than one kit used. This might underestimate the incidence of infection; however, while the incidence seen in our review may be lower than the actual incidence, it still falls well within the reported range for ventriculostomy related infections.

Data for this retrospective cohort study came from three related but different sources (microbiological records, patient charts, and hospital inventories for lumbar drain kits). We used the data from one source (the patient chart) to cross check the others. Data from the microbiology laboratory were used to select the study cohort.

This is by contrast with identifying patients with lumbar drains and reviewing those records to calculate an infection rate. A prospective study could identify patients in whom meningitis was thought to have existed (either on clinical grounds or CSF findings) and treatment instituted even in the absence of positive cultures or where organisms were seen on CSF examination and the cultures failed to grow. Many patients undergoing lumbar drainage receive antibiotic therapy for other reasons (as in the present study), possibly giving falsely negative cultures in the face of true CSF infection.

\section{Conclusions}

Continual external lumbar CSF drainage seems to carry a low risk of culture proved bacterial meningitis $(4.2 \%)$. Lacking obstruction to CSF outflow, a lumbar drain may pose less infectious risk than a ventriculostomy. Skin organisms most commonly cause this infection, which often appears within 24 hours of drain placement. Concomitant antibiotic use does not seem to offer protection; however, we examined the concurrent use of antibiotics as a group of agents and not the particular agent used. Thus, we cannot assert that what protection, if any, antibiotics may offer; such a determination awaits prospective evaluation. From our findings, we suggest: (1) Using a sterile technique, lumbar 
drains can be safely placed and maintained both in and out of acute care units (the intensive care unit); and (2) as the presence of leukocytosis, fever, and pleocytosis may have little predictive ability in heralding the appearance of lumbar drain associated bacterial meningitis, this complication of lumbar drainage should be firmly diagnosed from routine CSF culture. Our findings support the contention that the presence of pleocytosis alone is insufficient and not specific for the diagnosis of lumbar drain associated bacterial meningitis; however, increasing CSF cell counts may offer some information valuable for assessing management strategies. Casecontrol and prospective observational studies could clarify better potential risk factors for contracting lumbar drain associated bacterial meningitis.

This work was supported in part by National Institutes of Health Grant No NS-30305 (WMC, HRW). We thank Kevin Anstrom, for assistance with statistical analyses, Heather $M$ Bybee, Dolors T Jones, Rosemary L Dolph, neurological surgery research nurses, for their assistance in identification of cases, and the clinical laboratory, especially the microbiology section, at Harborview Medical Center for its assistance in clinical laboratory data collection.

1 Weir B. Physiology and pharmacology of aneurysmal rupture. In: Aneurysms affecting the nervous system. Baltimore:Williams and Wilkins, 1987:287.

2 Sato J, Sato O, Kamitani $\mathrm{H}$, et al. Intracranial surgery and postoperative management of patients with ruptured aneurysm in acute and subacute stage. Basal cisternal drainage and lumbar subarachnoid drainage. Neurol Med Chir 1979 19:173-9.

3 Mayhall CG, Archer NH, Lamb VA, et al. Ventriculostomyrelated infections. A prospective epidemiologic study. $N$ Engl F Med 1984;310:553-9.

4 Raquet F, Mann WJ. Lumbar cerebrospinal fluid drainage for prevention of cerebrospinal fluid fistulas. HNO for prevention

5 Huang CI, Huang MC, Chen IH, et al. Diverse applications of continuous lumbar drainage of cerebrospinal fluid in neurosurgical patients. Ann Acad Med Singapore 1993, 22(suppl 3):456-8.

6 Kusske JA, Turner PT, Ojemann GA, et al. Ventriculostomy for the treatment of acute hydrocephalus following subarachnoid hemorrhage. F Neurosurg 1973;38:591-5.

7 Lundberg N, Troupp H, Lorin H. Continuous recording of the ventricular-fluid pressure in patient with severe acute traumatic brain injury. A preliminary report. $\mathcal{f}$ Neurosurg 1965;22:581-90.
8 Smith RW, Alksne JF. Infections complicating the use of external ventriculostomy. F Neurosurg 1976;44:567-70.

9 Bering EA Jr. A simplified apparatus for constant ventricular drainage. $\mathcal{F}$ Neurosurg 1951;8:450-2.

10 Kim DK, Uttley D, Bell BA, et al. Comparison of rates of infection of two methods of emergency ventricular drainage. $\mathcal{F}$ Neurol Neurosurg Psychiatry 1995;58:444-6.

11 Wyler AR, Kelly WA. Use of antibiotics with external ventriculostomies. $\mathcal{F}$ Neurosurg 1972;37:185-7.

12 Sundbarg G, Kjallquist A, Lundberg N, et al. Complications due to prolonged ventricular fluid pressure recording in clinical practice. In: Intracranial pressure: experimental and clinical aspects. Berlin:Springer-Verlag, 1972:348-52.

13 Lundberg N. Continuous recording and control of ventricular fluid pressure in neurosurgical practice. Acta Psychiatr Scand 1960;149(suppl):1-193.

14 Hasan D, Vermeulen M, Wijdicks EFM, et al. Management problems in acute hydrocephalus after subarachnoid hemorrhage. Stroke 1989;20:747-53.

15 Hasan D, Lindsay KW, Vermeulen M. Treatment of acute hydrocephalus after subarachnoid hemorrhage with serial lumbar puncture. Stroke 1991;22:190-4.

16 Khanna RK, Rosenblum ML, Rock JP, et al. Prolonged external ventricular drainage with percutaneous longtunnel ventriculostomies. F Neurosurg 1995;83:791-4.

17 Amsel S, Fried D. Pneumococcal meningitis following lumbar puncture. Harefuah 1983;105:264-5.

18 Bergman I, Wald ER, Meyer JD, et al. Epidural abscess and vertebral osteomyelitis following serial lumbar punctures. Pediatrics 1983;72:476-80.

19 Bocker H, Franek A. Meningitis following lumbar puncture [letter]. Dtsch Med Wochenschr 1987;112:120.

20 Domingo P, Mancebo J, Blanch L, et al. Iatrogenic streptococcal meningitis [letter]. Clin Infect Dis 1994;19:356-7.

21 Helwig H. Meningitis following lumbar puncture [letter] Dtsch Med Wochenschr 1986;111:1461.

22 Lanska DJ, Lanska MJ, Selman WR. Meningitis following spinal puncture in a patient with a CSF leak. Neurology 1989;39:306-7.

23 Smith KM, Deddish RB, Ogata ES. Meningitis associated with serial lumbar punctures and post-hemorrhagic hydrocephalus. F Pediatr 1986;109:1057-60.

24 Torres E, Alba D, Frank A, et al. Iatrogenic meningitis due to Streptococcus salivarius following a spinal tap [letter]. Clin Infect Dis 1993;17:525-6.

25 Torres-Morera LM, Caballero-S'anchez V, Alonso-Blanco $\mathrm{F}$, et al. Septic meningitis following intradural anesthesia. Rev Esp Anestesiol Reanim 1988;35:46-8.

26 Bissagnene E, Kouassi YF, Ba ZV, et al. Streptococcus pneumoniae meningitis after myelography and intrathecal infiltration. Bull Soc Pathol Exot Filiales 1989;82:572-4.

27 Morparia HK, Vontivillu J. Case report: cerebrospinal fluid fistula. A rare complication of myelography. Clin Radiol 1991;44:205.

28 Dimitriu SM, Luca V, Turcu T, et al. Acute nosocomial bacterial meningitis. Apropos 27 cases. Rev Med Chir Soc Med Nat Iasi 1992;96:19-25.

29 Eng RH, Seligman SJ. Lumbar puncture-induced meningitis. $\mathcal{F A M A ~ 1 9 8 1 ; 2 4 5 : 1 4 5 6 - 9 .}$ 